\title{
Structural Characterization of Nano-Oxide Layers in PtMn Based Specular Spin Valve Thin Films
}

\author{
Lifan Chen
}

The Center for Nanoscale Science and Technology, Rice University, MS-366, 6100 Main Street, Houston, TX 77005, USA

In order to meet the requirements of magnetic read heads for higher density recording, a larger magnetoresistance for current spin valves is needed. One of the promising ways to increase the giant magnetoresistance (GMR) is the use of specular reflection layers such as nano-oxide layers (NOLs) [1-2]. Although NOLs play an important role to enhance GMR effect in specular spin valves, the process control of the NOLs still remains a challenge for the applications in disk drives. The relationship between NOL structure and magnetic correlation is still not fully understood so far [3]. Therefore, it is necessary to understand the structural charactorization of NOLs in spin valves at atomic level.

The PtMn based specular spin valve structures studied in this paper are $\mathrm{Ta} / \mathrm{NiFeCr} / \mathrm{PtMn} /$ $\mathrm{CoFe} / \mathrm{Ru} / \mathrm{CoFe} / \mathrm{NOL} / \mathrm{CoFe} / \mathrm{Cu} / \mathrm{CoFe} / \mathrm{NiFe} / \mathrm{Cu} / \mathrm{Ta}-\mathrm{O}$. All samples were deposited with magnetron sputtering and annealed at $230-300^{\circ} \mathrm{C}$ under an in-plane magnetic field in a vacuum furnace for 8-10 hours. The NOLs at pinned layer side were fabricated by oxidizing CoFe synthetic pinned layer $\mathrm{CoFe} / \mathrm{Ru} / \mathrm{CoFe} / \mathrm{NOL} / \mathrm{CoFe}$ with natural and plasma oxidation. The oxygen exposure was controlled to be below $10 \mathrm{mT} \cdot \mathrm{sec}$ in both oxidation processes. A systematic structure characterization of nanooxide layers and specular spin valves by using high-resolution transmission electron microscopy (HRTEM) has been studied.

Figure 1 and 2 show that the specular reflection effect from the NOLs was found to enhance GMR ratio from $12 \%$ in the spin valve with no NOL to more than $15 \%$ and $16 \%$ in the case of natural and plasma oxidation of the NOL, an increase in GMR ratio by more than 30\%. Oxygen exposure plays an important role in NOLs process. HRTEM reveals that oxide clusters mixing with insufficiently oxidized CoFe layers prevailed in natural NOL, and the natural oxidation was inhomogeneous, as shown in Figure 3. In contrast, plasma NOL has a thinner, more homogeneously oxidized CoFe layers with sharp interfaces, as shown in Figure 4. In plasma NOLs, the structures still maintain CoFe crystal structure. This comparison indicates that plasma oxidation is much better than natural oxidation to create desirable NOLs. A large GMR ratio can be obtained in NOL specular spin valves by plasma oxidation process. Fabricating NOLs without any degradation in crystal structure is critical to obtain high GMR ratio. In plasma NOLs, CoFe fcc crystal structure with minimum crystal degradation have been achieved.

\section{References}

[1] W. F. Egelhoff, Jr., P. J. Chen, C. J. Powell, M. D. Stiles, R. D. McMichael, J. H. Judy, K. Takano, and A. E. Berkowitz, J. Appl. Phys. 82 (1997) 6142.

[2] W. F. Egelhoff, Jr., P. J. Chen, C. J. Powell, D. Parks, G. Serpa, R. D. McMichael, D. Martien, and A. E. Berkowitz, J. Vac. Sci. Technol. B17 (1999) 1702. 
[3] J. Hong, K. Noma, and H. Kanai, J. Appl. Phys. 89 (2001) 6940.

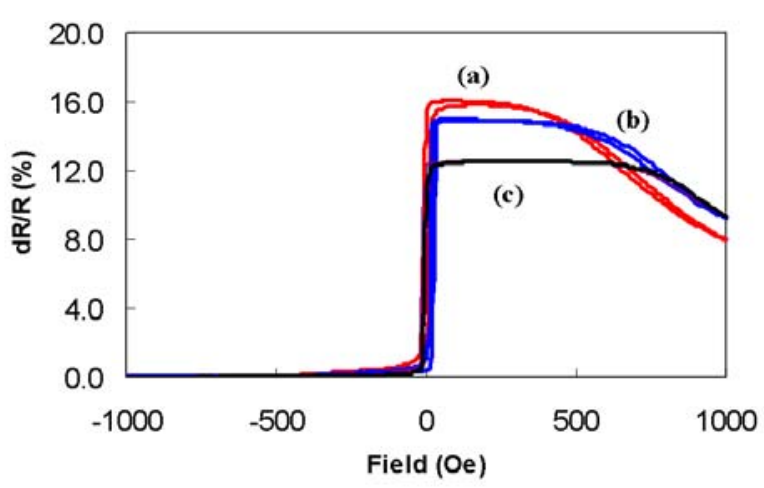

Fig.1 GMR curves for spin valves with (a) plasma oxidized NOL (b) natural oxidized NOL (c) non-NOL in the pinned layer, with $\mathrm{dR} / \mathrm{R}=16 \%, 15 \%$ and $12 \%$, respectively.

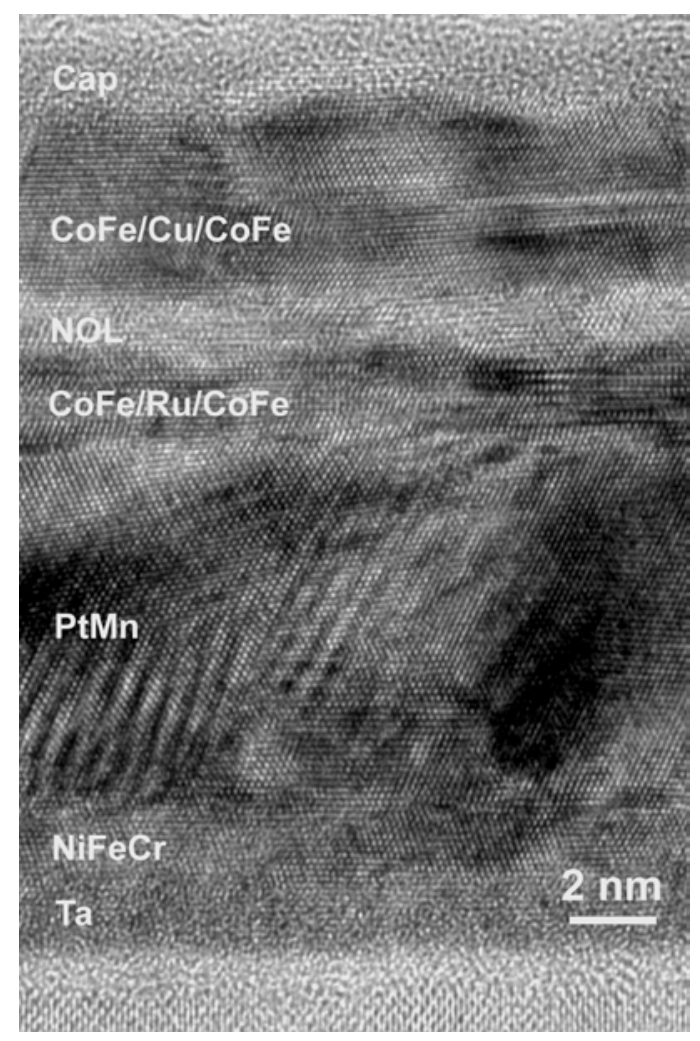

Fig. 3. Cross-sectional HRTEM of specular spin valves with NOL prepared by natural oxidation process.

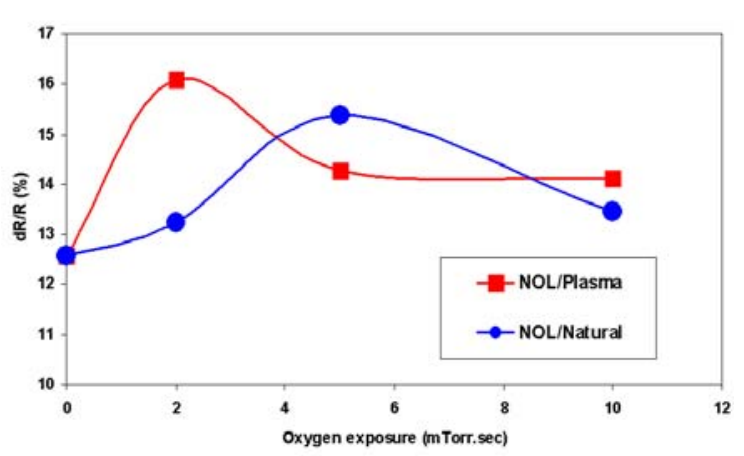

Fig. 2. GMR ratio as a function of oxygen exposure for natural and plasma oxidation processes.

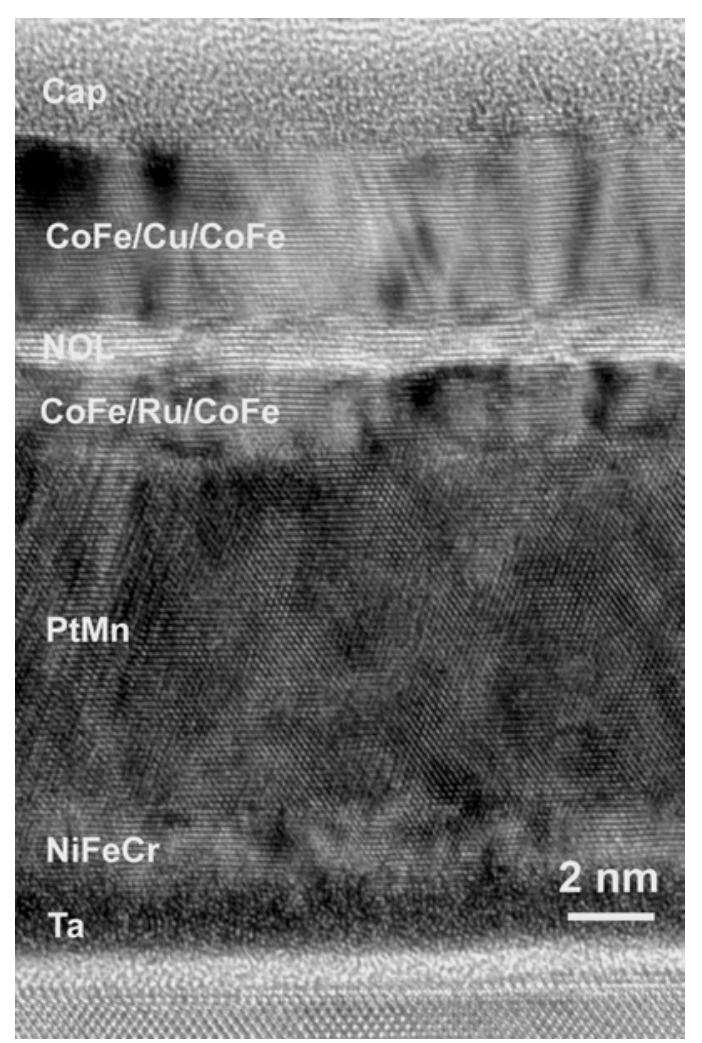

Fig. 4. Cross-sectional HRTEM of specular spin valves with NOL prepared by plasma oxidation process. 